\title{
Luminal B Breast Carcinoma
}

National Cancer Institute

\section{Source}

National Cancer Institute. Luminal B Breast Carcinoma. NCI Thesaurus. Code C53555.

A biologic subset of breast carcinoma defined by low to moderate expression of genes

characteristic of luminal epithelial cells including estrogen receptor (ER), and high expression of GGH, LAPTM4B, and CCNE1. This subtype of breast cancer is associated with a good prognosis, although not as favorable as the luminal A subtype. 